ALICE/PUB 97-01

23 January 1997

\title{
LAYOUT OF THE ALICE EXPERIMENT AT THE LHC
}

\author{
W. Klempt and G. Paić \\ CERN, Geneva, Switzerland \\ for the ALICE Collaboration
}

\begin{abstract}
A short description is given of the ALICE experiment, dedicated to the study of heavy-ion collisions at the LHC. The design and performance of the main detectors are presented as well as some $R \& D$ results.
\end{abstract}

To be published in the Proceedings of the 5th International Conference on Advanced Technology and Particle Physics, Como, Italy, 7-11 October 1996.

(Nuclear Physics B) 
ALICE (A Large Ion Collider Experiment) [1, 2] is the only LHC experiment fully dedicated to the study of heavy-ion collisions at a momentum of $\sim 2.76 \mathrm{TeV} / c$ per nucleon and beam. The prime aim of the detector is to study in detail the behaviour of matter at high energy densities in order to establish the existence of QCD bulk matter and to bring into evidence the characteristics of the phase transition from hadronic to the quark-gluon plasma.

The detector has the very complex task of covering almost all the hadronic and leptonic signals using a single set-up, a situation very different from the one prevailing today at the SPS with seven experiments, each running with a well-defined optimization for specific signals, and the one at the Relativistic Heavy Ion Collider (RHIC) in construction at Brookhaven with four running experiments with clearly separate tasks in the hadronic and leptonic sector. The detector has to respect constraints that can be summarized as follows:

- Multiplicity of the events

The multiplicity of charged particles currently predicted in the midrapidity region of the ALICE detector varies widely from generator to generator, from $(\mathrm{dN} / \mathrm{d} \eta)_{\mathrm{ch}} \sim 1400$ for the String Fusion Model with fusion, to 7000 per pseudorapidity unit for the VENUS4.12 generator [3]. In the conception of the experiment a rapidity density of 8000 per unit has been used. The high multiplicity drives the granularity of the detectors, and the optimal distance of each of them from the colliding beams.

- The momenta and masses to be measured

The study of ion-ion collisions means that particles need to be measured in a large range of momenta and masses. The momenta we propose to measure range between $60 \mathrm{MeV}$ and $10 \mathrm{GeV} / c$ in the central barrel. The range of masses, detected and identified, goes from electrons to upsilons. These requirements govern the rather complex particle identification scheme, and the low magnetic field adopted.

- Rate of acquisition

The rate of interaction in lead-lead collisions is about $8000 \mathrm{~Hz}$ with approximately $10 \%$ of central events. This relatively low rate of interaction-compared with the rate of the pp experiments at the LHC - allows us to use in the set-up a large palette of detectors, from those with fast response to intrinsically slow detectors like the time projection chamber. We shall review the present design as in the Technical Proposal and the Addendum to the Technical Proposal $[1,2]$ for the ALICE experiment. In our description we shall concentrate on the main characteristics of the detectors with reference to the developments in progress, and especially to those that, coupled with ongoing experiments with heavy ions at the SPS, point to the feasibility and robustness of the design.

\section{DETECTOR OVERVIEW}

The overall layout of the ALICE experiment is shown in Fig. 1. The detector consists of two parts: the central one (centred around midrapidity) and the forward part with the muon spectrometer, the forward multiplicity detectors, and the zero-degree calorimeter. 


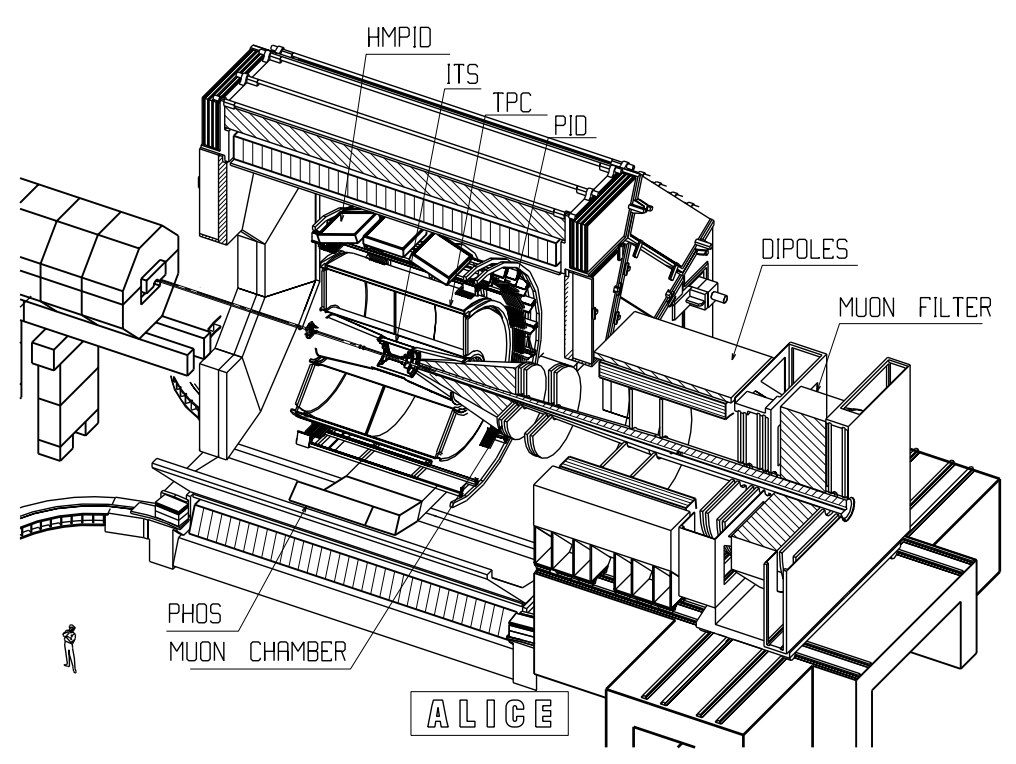

Figure 1: Layout of the ALICE detector.

\section{LAYOUT OF THE CENTRAL PART}

The central part of the ALICE detector is devoted to the detection of hadronic signals: momentum spectra, particle ratios, measurement of the space-time evolution of the system after collision through the use of particle interferometry. The central part, which covers $\pm 45^{\circ}$ over the full azimuth, is embedded in the existing LEP L3 magnet with a weak solenoidal field of $0.2 \mathrm{~T}$. It consists, starting from the interaction point, of an Inner Tracking System (ITS) with six layers of high-resolution silicon tracking detectors, a cylindrical TPC, and a large-area particle identification array of time-of-flight (TOF) counters. In addition, there are two small-area, single-arm detectors: an electromagnetic calorimeter (PHOS) and an array of counters optimized for high-momentum inclusive particle identification (HMPID).

\subsection{Inner tracker (ITS)}

The purpose of the Inner Tracking System is to detect the vertices of decays of neutral particles decaying close to the interaction point (hyperons and charmed particles) as well as to extend the useful range of momentum to the lowest possible limit. To satisfy these requirements the system has six cylindrical layers of coordinate sensitive detectors. The large multiplicities encountered, and the precision on the secondary vertices have dictated the use of different types of detectors. The two innermost layers have thus been designed using Silicon Pixel Detectors (SPD). The next two layers consist of Silicon Drift Detectors (SDD), followed by two layers of Silicon Strip Detectors (SSD). For reasons of particle identification at low momenta, and more efficient tracking all but the pixel layers will have analog readout.

The main parameters for the silicon detector of the ITS are shown in Table 1.

\subsubsection{Silicon pixel layer (SPD)}

Extensive R\&D is in progress to develop a silicon pixel detector, $81 \mathrm{~mm}$ long, $13.3 \mathrm{~mm}$ wide and $0.15 \mathrm{~mm}$ thick, with $256 \times 256$ cells of $50 \times 300 \mu \mathrm{m}$ bump-bonded to the readout chips. A chip of similar design is being used in the WA97 experiment with a somewhat larger cell size $(50 \times 500 \mu \mathrm{m}$ in a $1 \mu \mathrm{m}$ SACMOS process $)$. A telescope using 
this type of pixels has been successfully used in the 1996 ion run for the study of $\mathrm{Pb}-\mathrm{Pb}$ collisions. In Fig. 2 we show a $\mathrm{Pb}-\mathrm{Pb}$ event with 153 tracks reconstructed in the pixel telescope.

Table 1

Parameters of the various detector types. A module represents a single detector.

\begin{tabular}{|c|c|c|c|c|c|c|c|c|c|c|}
\hline \multirow[t]{3}{*}{ Туре } & \multirow{2}{*}{\multicolumn{2}{|c|}{$\begin{array}{c}\text { Spatial } \\
\text { precision } \\
(\mu \mathrm{m})\end{array}$}} & \multirow{2}{*}{\multicolumn{2}{|c|}{$\begin{array}{c}\text { Two-track } \\
\text { resolution } \\
(\mu \mathrm{m})\end{array}$}} & \multirow{3}{*}{$\begin{array}{l}\text { Cell } \\
\text { size } \\
(\mu \mathrm{m})\end{array}$} & \multirow{3}{*}{$\begin{array}{l}\text { Module } \\
\text { size } \\
(\mathrm{mm})\end{array}$} & \multirow{3}{*}{$\begin{array}{c}\text { Channels } \\
\text { per } \\
\text { module }\end{array}$} & \multirow{3}{*}{$\begin{array}{l}\text { Electr. } \\
\text { channels } \\
\text { total } \\
(\mathrm{k})\end{array}$} & \multicolumn{2}{|c|}{$\begin{array}{c}\text { Power } \\
\text { dissipated }\end{array}$} \\
\hline & & & & & & & & & Barrel & End-caps \\
\hline & $r \varphi$ & $z$ & $r \varphi$ & $z$ & & & & & $(\mathrm{~W})$ & $(\mathrm{W})$ \\
\hline Pixel & 15 & 90 & 100 & 600 & $50 \times 300$ & $13.3 \times 81$ & 65536 & 14420 & 450 & - \\
\hline Drift & 20 & 30 & 200 & 420 & - & $70 \times 87$ & $2 \times 384$ & 192 & 350 & 1950 \\
\hline Si strip & 30 & 860 & 200 & 7000 & $95 \times 41000$ & $75 \times 42$ & $2 \times 768$ & 2620 & $<3000$ & $<1000$ \\
\hline
\end{tabular}

153 tracks through the Si Pixel Telescope

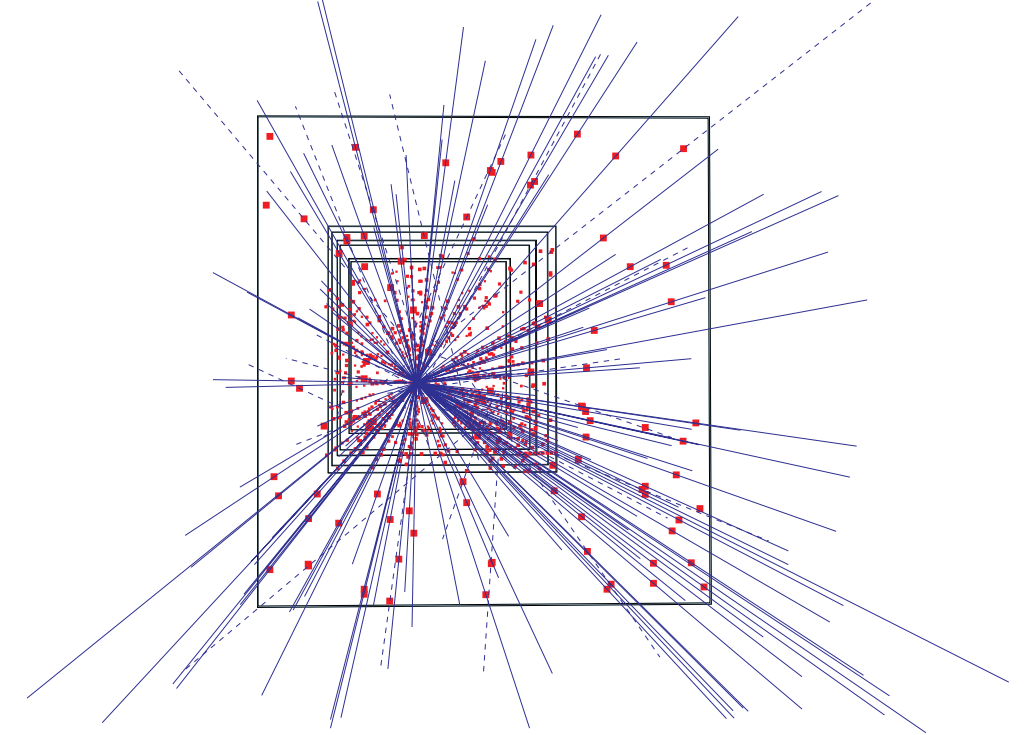

Figure 2. Tracks reconstructed from a $\mathrm{Pb}-\mathrm{Pb}$ collision in the WA97 pixel telescope.

\subsubsection{Silicon drift layers (SDD)}

Efforts are being made to develop a design suitable for production by industry, along the lines of already operating or planned detectors (STAR at RHIC). A design in which the sensitive area is split in two parts is being considered. Such a design allows the electrons to drift from the centre to two anodes, thus reducing the drift length by two compared with a design where the anode array is on one side of the detector. Early prototypes of the full-size ALICE module $(70 \times 87 \mathrm{~mm})$ are now being tested. Each detector has 384 anode pads on each edge, with a pitch of $210 \mu \mathrm{m}$. A linear resistor chain is integrated either side onto the detector chip and defines the potential of each strip and thus the drift field. Figure 3 shows for all strips the measured potential deviation as compared to an ideal divider chain for both the $\mathrm{p}$ - and n-side of the detector in the case of a smaller prototype. The measured deviation is compatible with the requirements for spatial resolution. The total dead area will be kept below 14\%. The simulations of the full detector-including electronic noise - foresee an average resolution for ALICE central events of $20 \mu \mathrm{m}$ in the drift direction (which is the relevant coordinate for momentum resolution) and $31 \mu \mathrm{m}$ in 
the transverse direction. The SDDs will be mounted on linear structures called ladders where care will be taken to overlap the detectors in order to fully cover the acceptance.

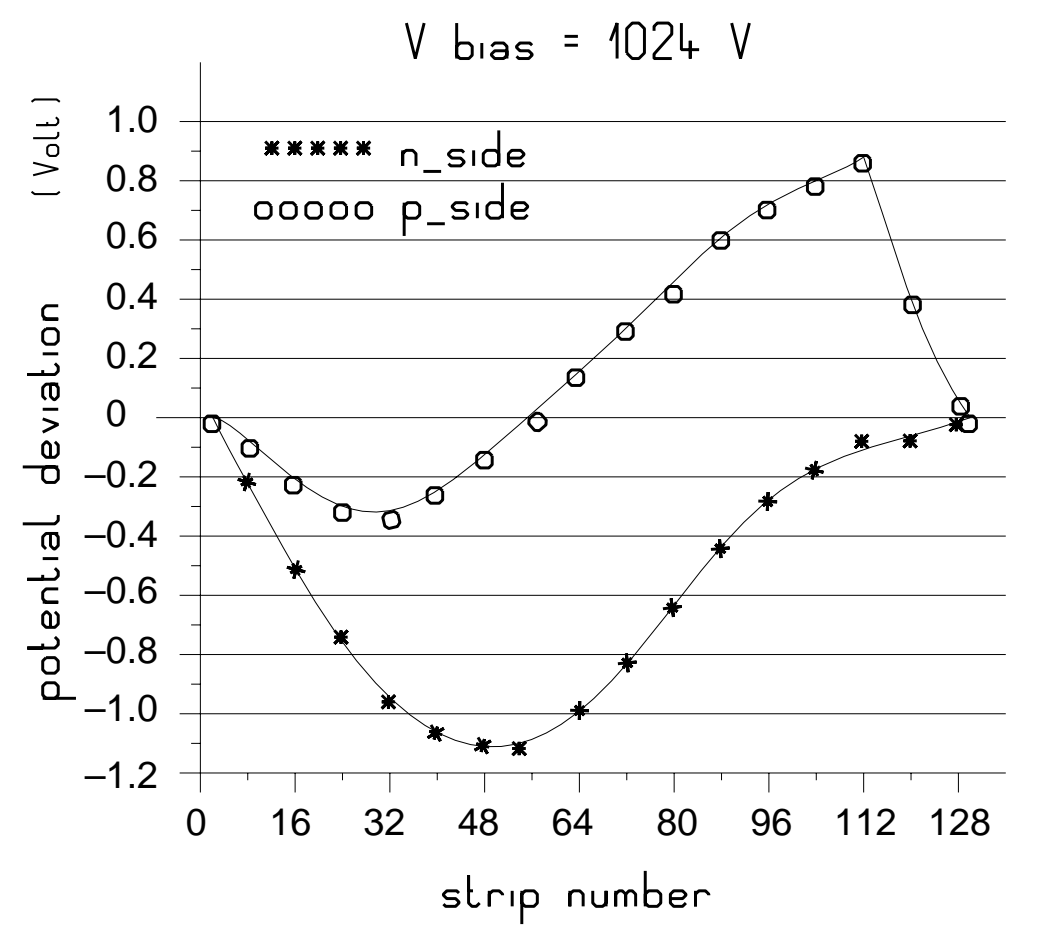

Figure 3. Deviation from linear potential distribution for each strip on the p- and n-side of the prototype silicon drift detector.

\subsubsection{Silicon strip layers (SSD)}

Silicon strip detectors are the most widely used silicon detectors in HEP experiments, available commercially in a large number of designs. The silicon strip layers of the ITS consist of double-sided SSDs with a stereo angle of $35 \mathrm{mrad}$ between the readout strips on either side of a single silicon wafer. The strips are oriented parallel to the beam line resulting in a resolution of $27 \mu \mathrm{m}$ for a $95 \mu \mathrm{m}$ pitch of the strips, and a resolution of $860 \mu \mathrm{m}$ along the beam direction. The detector thickness is $300 \mu \mathrm{m}$ of silicon, representing $\sim 0.3 \%$ of a radiation length. The other components (electronics, cabling, cooling) increase the thickness to $\sim 0.5 \%$. The size of the layer and the requirements of position precision necessitate a very rigid frame for the SSD layers. A special carbon-fibre spaceframe was designed and built. The front-end analog electronics will have a 128-channel preamplifier shaper circuit equipped with sample/hold, multiplicity-measuring capability with a power consumption of $1 \mathrm{~mW} /$ channel.

\subsection{Time Projection Chamber (TPC)}

The TPC is the main tracking detector of ALICE. Beyond tracking and momentum determination in conjunction with the ITS, the TPC is also required to provide particle identification in the range of momenta where $\mathrm{d} E / \mathrm{d} x$ particle identification is possible. The TPCs have been the workhorses of many HEP experiments, and lately the NA49 experiment has demonstrated their unimpaired performance under extreme conditions of particle multiplicities. The TPC is an inherently slow detector requiring about $100 \mu \mathrm{s}$ drift time over the $2.5 \mathrm{~m}$ length from the central plane to one end of the detector. However, it can cope with the $10 \mathrm{kHz}$ minimum-bias rate expected at the design luminosity for lead ions at the $\mathrm{LHC}$ of $10^{27} \mathrm{~cm}^{-2} \mathrm{~s}^{-1}$. 
The TPC has a cylindrical shape. The electric drift field is constant $(\sim 400 \mathrm{~V} / \mathrm{cm}$ resulting in a drift velocity of $2.5 \mathrm{~cm} / \mu \mathrm{s}$ and diffusion constants of $\sim 250 \mu \mathrm{m} / \sqrt{\mathrm{cm}}$ ) and points from the central HV plane towards the two readout planes at the end-caps. The TPC will use a Ne-based gas mixture. The inner radius is at $0.88 \mathrm{~m}$ from the beam axis, corresponding to particle densities of $0.1-0.2$ particles $/ \mathrm{cm}^{2}$. Such densities are known, from the experience of NA49, to allow efficient tracking and $\mathrm{d} E / \mathrm{d} x$ measurements. The outer radius has been chosen to be $2.5 \mathrm{~m}$ to achieve sufficient track length for a good momentum resolution and to achieve a $\mathrm{d} E / \mathrm{d} x$ resolution of the order of $7 \%$. Recently simulations have shown [4] that for 75 samples per track, taking a truncated mean ionization using the 45 lowest value points, one can achieve such a resolution. It is comforting that the results of the present simulation are in complete agreement with the experimental $\mathrm{d} E / \mathrm{d} x$ resolution achieved in the NA49 TPC for a similar particle density, if one allows for the differences in track length and number of samples. The experimental results [5] obtained for 90 samples are shown in Fig. 4.

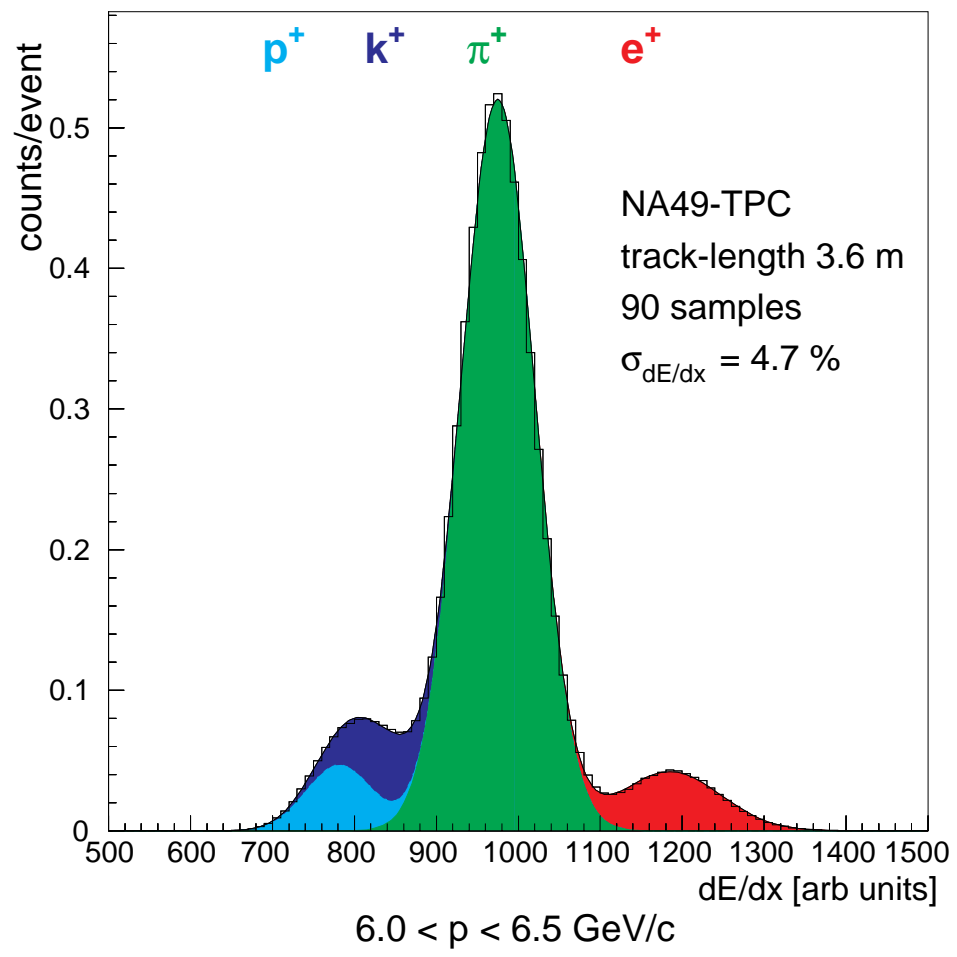

Figure 4. Ionization loss spectra obtained in the NA49 $\mathrm{TPC}$ in $\mathrm{Pb}-\mathrm{Pb}$ collisions at $168 \mathrm{~A} \mathrm{GeV} / c$.

\subsection{Hadron identification above $700 \mathrm{MeV} / c$}

Particle identification (PID) at momenta above those attainable by $\mathrm{d} E / \mathrm{d} x$ in the ITS and TPC is an important design feature of the ALICE detector. The requirements on the PID performance depend on the planned physics, hence we can clearly identify two categories:

- Event-by-event hadron identification

The large multiplicities encountered in the collisions offer an interesting opportunity to extract statistically significant information on some global parameters from single events (slopes of spectra, particle ratios, source sizes, etc.). Because of the steeply falling slopes of momentum spectra there is a limit where the number of particles becomes statistically insignificant on an event-by-event basis. 
This limit is estimated to be $1.5-2 \mathrm{GeV} / c$ for ALICE conditions for a PID covering the same phase space as the other barrel detectors like the ITS and TPC.

At present two alternative solutions are under consideration: the baseline design relies on a Pestov counter technology [6] with a time resolution $\leq 100 \mathrm{ps}$, while the Parallel Plate Chambers (PPC) [7] represent a fallback solution, albeit with a time resolution of only $\sim 150-200$ ps.

- Inclusive hadron identification

The possibility to select on an event-by-event basis "classes" of events of similar properties means that one can conduct "semi-inclusive" analysis of such subsamples enlarging thus the number of measurable observables to those not statistically significant in single events (hyperon decays, hard part of momentum spectra etc.). These considerations, to identify hadrons in the hardest part of the spectrum (i.e. above $1.5-2 \mathrm{GeV} / c$ ), warrant the need for a PID detector performing above the capability of the barrel PID but in a small part of the phase space. This high-momentum PID (HMPID) covers about 5\% of the phase space of the central barrel. The baseline design for this detector is a proximity focusing $\mathrm{RICH}$ detector with a liquid radiator.

\subsubsection{The Pestov counter TOF}

The Pestov spark counter is a single-gap, gaseous, parallel-plate detector working in the streamer/spark mode. The ALICE design considers a strip geometry module with "cells" consisting of two adjacent strips of $30 \mathrm{~cm}$ length and $2.54 \mathrm{~mm}$ width corresponding to an occupancy of $\sim 10 \%$ at the highest multiplicities predicted in ALICE. The spark gap is $100 \mu \mathrm{m}$. The strips are read out at both ends in order to obtain information on the position of the hit along the strip with a precision of $\sim 1 \mathrm{~mm}$. The TOF barrel will have in total 95000 cells with 380000 electronic readout channels (each cell consisting of two strips read out at both ends). In the past few years considerable progress has been made in mastering the technological problems linked to the operation, ageing, and mass production of these counters. Time resolutions of $32 \mathrm{ps}$ have been achieved in prototypes [8].

\subsubsection{Parallel Plate Chambers (PPC)}

A PPC is a gaseous detector working in the avalanche mode [7]. It consists of planar metallized electrodes made of ceramic or plastic kept at a distance of 1-2 mm by spacers. The ALICE collaboration plans to use a double gas gap design enhancing thus the efficiency of the detector, and possibly the time resolution. The current developments indicate that excellent intrinsic detection efficiency can be obtained with resolutions of $\sim 190$ ps. This modest time resolution can be improved using a two-layer design where two independent time measurements are performed for each particle.

\subsubsection{TOF barrel performances}

In Fig. 5 we show the efficiency of identification as well as the level of contamination for the main particle species. The performance has been calculated taking into account the time resolution (100 ps), the matching efficiency for tracks from the TPC to the TOF barrel, and the noise hits created by secondary particles [9].

\subsubsection{High-Momentum PID by RICH (HMPID)}

The HMPID with a coverage of $\sim 5 \%$ of the phase space identifies hadrons beyond the capability of the PID barrel. The surface of the detector has been determined by 
the requirement for the detection of high-momentum hadronic pairs for interferometry purposes.

The RICH HMPID array has a total area of $\sim 12 \mathrm{~m}^{2}$ located at $4.7 \mathrm{~m}$ from the beam to benefit from the lowest particle density $\left(\leq 50\right.$ particles $\left./ \mathrm{m}^{2}\right)$.

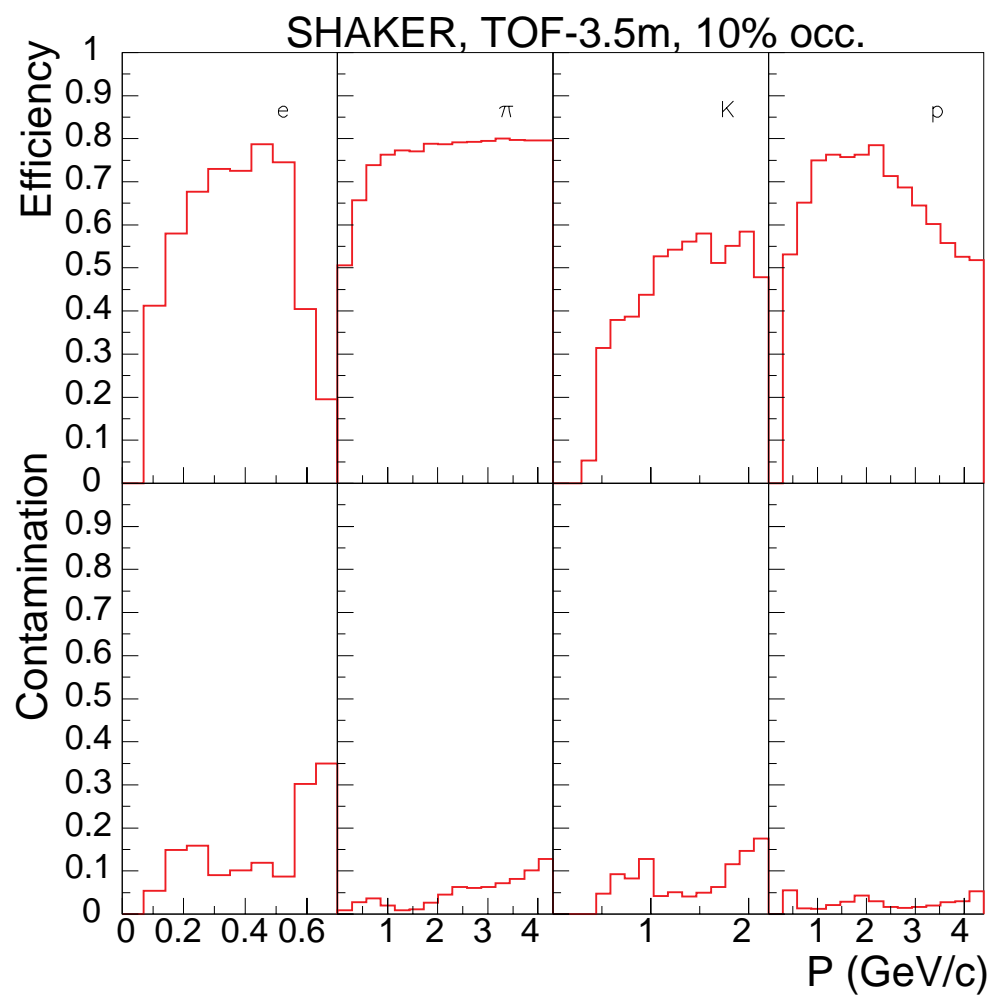

Figure 5. Efficiency of identification (upper part) and corresponding contamination (lower part) for different species as a function of momentum.

The $\mathrm{RICH}$ detector consists of a $\mathrm{C}_{6} \mathrm{~F}_{14}$ radiator with good transparency in high UV (until $165 \mathrm{~nm}$ ) contained with a quartz window towards the photon detector located $\sim 7 \mathrm{~cm}$ away, in order to achieve sufficient angular resolution in the ring-like pattern of emitted photons on the photon detector plane. The photon detector uses recently developed CsI photocathodes, in a reflective mode, evaporated directly on the pad cathode substrate of a multiwire proportional chamber. The cathode's segmentation into $8 \times 8 \mathrm{~mm}^{2}$ pads allows satisfactory angular resolution. This technique, developed for large areas within the RD26 project at CERN, has resulted in good quantum efficiency using simple substrates consisting of printed-circuit boards with a prepolished copper layer subsequently covered with a thick nickel layer and a thin layer of chemical gold [10]. Beam tests indicated a good performance over several months of operation and no appreciable degradation after storage of up to two years, in a nitrogen atmosphere. The pattern recognition, performed using simulated events constructed from single-particle events measured with prototypes, indicates that in a dense environment a particle identification and contamination as shown in Fig. 6 can be achieved.

\subsection{Photon spectrometer (PHOS)}

The task of the PHOS is to search for direct photons emitted in the collisions and to measure neutral pions and etas at the largest momenta $(\sim 25 \mathrm{GeV} / c)$ where the momentum resolution in the spectrometer is better than for charged particles measured in the 
tracking system. The detection of such particles gives information about the propagation of jets in a dense medium and is therefore of importance.

The PHOS will have a charged-particle veto detector in front to eliminate charged particles reaching it. The spectrometer will cover $\sim 6 \%$ at a distance of $4.6 \mathrm{~m}$ from the beam. The PHOS is built of $\mathrm{PbWO}_{4}$ [11] crystals of the size $2.2 \times 2.2 \times 20 \mathrm{~cm}^{3}$.

For the light readout, PIN diodes are considered to be the best candidate, although alternative detectors like phototetrodes and photopentodes are also under consideration.

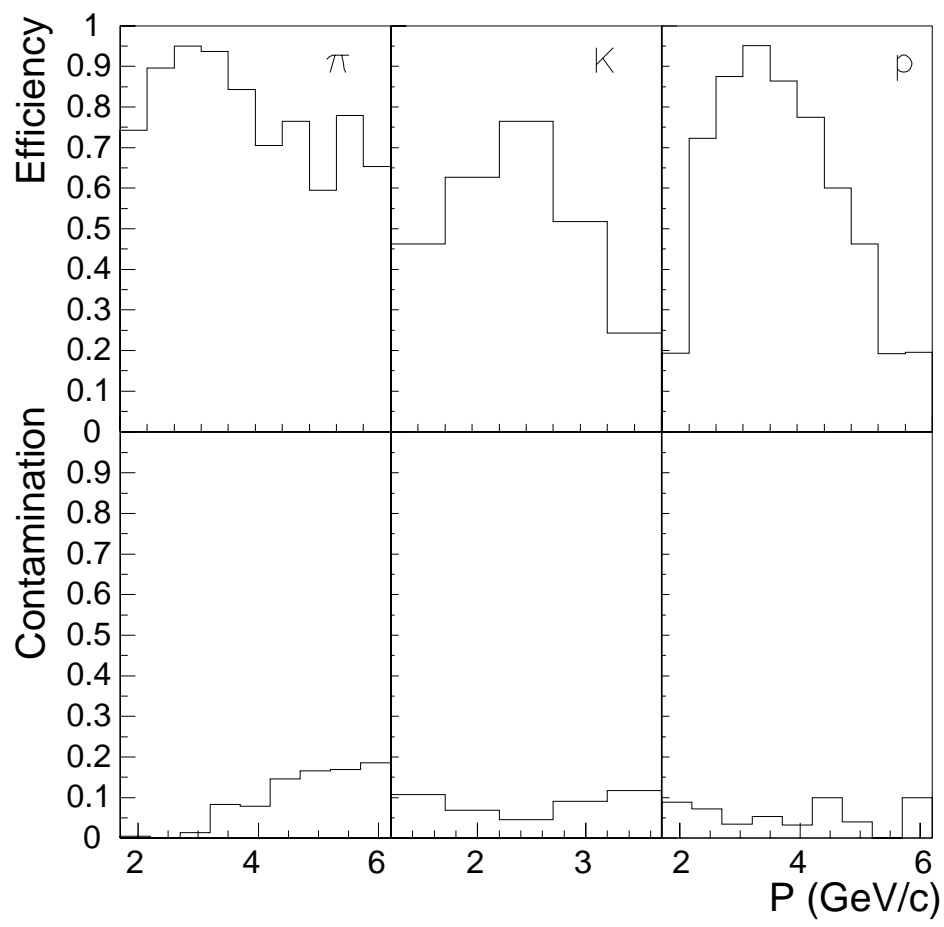

Figure 6. Efficiency of identification and corresponding contamination for pions, kaons, and protons in the HMPID.

\section{LAYOUT OF THE FORWARD MUON SPECTROMETER (FMS)}

While the central ALICE detector addresses mostly signals emitted in the late part of the collision (after hadronization), the forward muon spectrometer is devoted to a very important component of the study of very high energy density systems - the socalled hard penetrating probes. Present theoretical considerations view the measurement of heavy quarkonia as an essential tool to study the earliest and hottest stages of the collisions. The interest of these probes lies primarily in the fact that the behaviour of the resonant quarkonium states formed in the early stages is different, depending on the state of the surrounding matter at that time [12]. For deconfined matter-quark-gluon plasma - it is expected that the resonances formed would, to a large extent, "dissolve" on the way out from the collision volume while passage through hadronic matter would have a different pattern of decreasing the yield as a function of initial energy density. To verify these predictions the FMS will measure $\mathrm{J} / \Psi$ and $\Psi^{\prime}$ yields at low $p_{\mathrm{t}}$, as well as $\Upsilon, \Upsilon^{\prime}$ and $\Upsilon^{\prime \prime}$ yields via the detection of the muon-pair decays of these resonances.

The principal layout of the FMS is shown in Fig. 7. The angular acceptance of the muon spectrometer goes from $2^{\circ}$ to $9^{\circ}(\eta=2.5-4)$, the mass resolution will be $\leq 100 \mathrm{MeV}$ at quarkonia masses of $10 \mathrm{GeV}$ in order to separate all resonant states. It consists of a composite absorber $\left(\sim 10 \lambda_{\text {int }}\right)$, starting $90 \mathrm{~cm}$ from the vertex, a large dipole magnet with 
a $3 \mathrm{~T}$ m field integral placed outside the L3 magnet, and 10 planes of thin, high-granularity tracking stations.

A second absorber ( $\sim 7.2 \lambda_{\text {int }}$ of iron) at the end of the spectrometer and four more detector planes are used for muon identification and triggering. The spectrometer is shielded throughout its length by a dense absorber tube, of about $60 \mathrm{~cm}$ outer diameter, which surrounds the beam pipe.

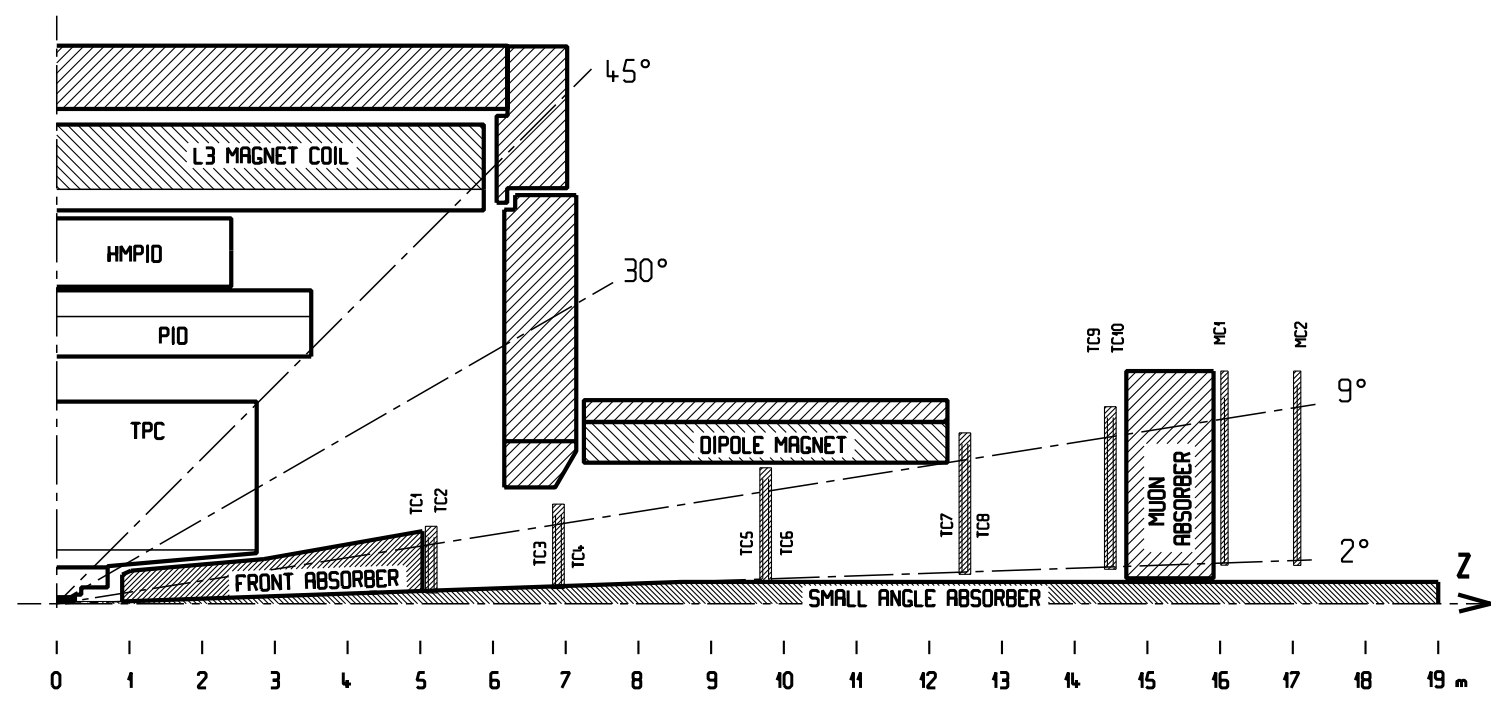

Figure 7. Schematic view of the dimuon arm.

\subsection{Absorbers}

- Front absorber

It is carefully optimized to keep the punchthrough into the muon tracking chambers and back-splash into the nearby TPC of the central barrel as small as possible. This has dictated a layered design consisting of dense low-Z materials (carbon and concrete) to limit multiple scattering in the front section, and a rear section containing alternating layers of neutron moderator and absorber (boronated polyethylene) and high-Z material (lead) to shield against neutrons and photons. Outside the muon arm acceptance a tungsten cone absorbs particles emanating from the beam pipe. On the outside of the absorber, lead tungsten and boronated polyethylene are used to reduce the particle load in the TPC.

- The beam shield

The small-angle beam shield is made of dense materials - pure tungsten in the most critical regions close to the interaction point and a cheaper tungsten-lead mixture elswhereencased in a $4 \mathrm{~cm}$ thick stainless-steel tube.

- The muon filter

The muon filter consists of a $5.6 \times 5.6 \times 1.2 \mathrm{~m}^{3}$ iron wall, located at $z=15 \mathrm{~m}$ between the last tracking and the first triggering plane.

\subsection{Tracking chambers}

The tracking chambers will be Cathode Strip Chambers (CSC), capable of achieving position resolutions of better than $100 \mu \mathrm{m}$. Two tracking chambers with two planes each are located in front of the muon magnet, two others behind, and one in its centre for a total of 10 tracking planes. Since the particle density decreases strongly from the centre to the outside of the chambers, the segmentation of the cathode plane is a function of particle density in order to keep a constant channel occupancy. 


\subsection{Trigger chambers}

The trigger chambers will be constructed with Resistive Plate Chambers, with four identical planes of $6 \times 6 \mathrm{~m}^{2}$ each. Each plane is subdivided into 14 individual RPCs read out on both cathodes with strips in the $x$ and $y$ directions. The size of the strips increases from the beam line, from 30 to $200 \mathrm{~cm}$ in length and from 1 to $3 \mathrm{~cm}$ in width, for a total of 20000 channels in the four trigger planes.

\section{CONCLUSION}

The LHC will provide colliding beams of heavy ions with a centre-of-mass energy of about $5.5 \mathrm{TeV} /$ nucleon. These collisions are expected to provide a very different, and significantly better environment for the study of strongly interacting matter than existing accelerators. ALICE, the only dedicated heavy-ion detector at LHC, will address most of the hadronic and leptonic signals of the quark-gluon plasma. The detector has been designed to access these signals in a conservative and robust way. The open geometry will facilitate future modifications and upgrades if first physics results suggest focusing on specific signals, selective, or larger acceptance.

\section{References}

[1] ALICE Collaboration, Technical Proposal, CERN/LHCC 95-71.

[2] ALICE Collaboration, Addendum to the Technical Proposal, CERN/LHCC 96-32.

[3] N. van Eijndhoven et al., Internal note ALICE 95-32.

[4] Marek Kowalski, Internal note ALICE 96-37.

[5] P. Seyboth, private communication.

[6] A.R. Frolov, Yu.N. Pestov and V.V. Primachek, Nucl. Instr. and Meth. A307 (1991) 497.

[7] Yu. Galaktionov et al., Nucl. Instr. and Meth. A317 (1992) 116.

[8] E. Badura et al., Internal note ALICE 96-19, and GSI preprint 96-22.

[9] S. Kiselev et al., Internal note ALICE 96-17.

[10] F. Piuz, Nucl. Instr. and Meth. A371 (1996) 96.

[11] P. Lecoq et al., CMS TN/94-308 (1994).

[12] H. Satz, Proc. Quark Matter 95, Nucl. Phys. A590 (1995) 63c. 